\title{
Multiscale Symbolic Transfer Entropy on Closing Eyes and Being Idle in $\delta$ rhythm of Electroencephalogram
}

\author{
Lizhao DU ${ }^{1, \text { a }}$, Wenpo $\mathrm{YAO}^{1, \mathrm{~b}}$, Xiaolin $\mathrm{HUANG}^{2, \mathrm{c}}$, Jun WANG ${ }^{1, \text { d,* }}$ \\ ${ }^{1}$ School of Geographic and Biologic information \\ Nanjing Univ. of Posts \& Telecomm \\ Nanjing, China \\ ${ }^{2}$ Institute of Biomedical Electronic Engineering \\ School of Electronic Science and Engineering \\ Nanjing University \\ Nanjing, 210093, China \\ E-mail: a18251955996@163.com \\ E-mail: byaowen512@163.com \\ E-mail: cxlhuang@nju.edu.cn \\ *E-mail: dwangj@njupt.edu.cn \\ +* Corresponding author
}

\begin{abstract}
As we all know, electroencephalogram (EEG) is an effective tool to analyze many disease, epilepsy, for example. According to the latest research, we have found out that the value of multiscale symbolic transfer entropy (MSTE) on closing eyes and being idle differs but there is a question still, what exactly makes this difference? Therefore, we analyze different rhythm of EEG and finally we draw this conclusion: the value of MSTE on closing eyes and being idle differs exactly in the $\delta$ rhythm of EEG. With this conclusion, we can catch the dynamic information much more convenient and accurately.
\end{abstract}

Keywords-multiscale symbolic transfer entropy(MSTE), Electroencephalogram (EEG), closing eyes, being idle

\section{INTRODUCTION}

Since electroencephalogram (EEG) is discovered in the 30 s in last century, we have learnt more and more about it in this nearly 80 years and even use it as a method to deal with diseases. Therefore, it is of great importance to learn more about EEG[1-5]. In this work, we analyze the value of multiscale symbolic transfer entropy (MSTE) on closing eyes and being idle in all four rhythms of EEG, and finally, we found out that it in the $\delta$ rhythm of EEG are definitely different. And this conclusion is useful for both health care and medical treatment. In this work, we first extract EEG from 12 persons when they are idle and closing their eyes respectively. Then, we use 5000 points and filtering it into different rhythm of EEG every time we analyze it. Next, we use MSTE, in other words, symbolize the filtered data firstly; and secondly calculate its (the result of first step) transfer entropy; lastly, multiscale it (the result of second step).

\section{METHODS}

\section{A. Filtering}

In our work, we use Chebyshev Type II filter to extract different rhythm of EEG. The four parameter required are passband corner frequency, stopband corner frequency, passband ripple and stopband attenuation. According to our research, we finally set the passband ripple and stopband attenuation as 10 and 50 respectively.

\section{B. Symbolization}

$$
\mathrm{S}_{\mathrm{i}}(\mathrm{i})\left\{\begin{array}{cc}
0: & \overline{\mathrm{x}}<\mathrm{x}_{\mathrm{i}+\mathrm{k}} \leq \overline{\mathrm{x}}+\alpha \times \mathrm{BS}(\mathrm{i}), \\
1: & \mathrm{x}_{\mathrm{i}+\mathrm{k}}>\overline{\mathrm{x}}+\alpha \times \mathrm{BS}(\mathrm{i}), \\
2: & \overline{\mathrm{x}}-\alpha \times \mathrm{BS}(\mathrm{i})<\mathrm{x}_{\mathrm{i}+\mathrm{k}} \leq \overline{\mathrm{x}}, \\
3: & \mathrm{x}_{\mathrm{i}+\mathrm{k}} \leq \overline{\mathrm{x}}-\alpha \times \mathrm{BS}(\mathrm{i}),
\end{array}\right.
$$

With the formula above [1-3], the original data turns into a symbolic sequence.

Here, $x_{i}$ is the $i^{\text {th }}$ point in the original data, $\bar{x}$ and BS (i) respectively denotes the mean and the base scale of the $\mathrm{i}^{\text {th }} \mathrm{m}$-dimensional vector $\mathrm{x}_{\mathrm{i}}$. According to our research, we set $\alpha$ as 0.05 .

\section{Transfer entropy}

After symbolizing, we then get a symbolic sequence like $1,2,3,3,2,1 \ldots$ We calculate how many times that every symbol has appears in this sequence. Last, we can calculate the transfer entropy[4] as follow Eq. 2.

$$
\operatorname{STE}_{X \rightarrow Y}=\sum p\left(x_{t+1}, x_{t}, y_{t}\right) \cdot \log \frac{p\left(x_{t+1}, x_{t}, y_{t}\right) p\left(x_{t}\right)}{p\left(x_{t}, y_{t}\right) p\left(x_{t}, x_{t+1}\right)}
$$

\section{Multiscale analysis}

With multiscale[5] ( as formula (3)), we divide the time series $x^{i}$ into a new one $y_{j}^{s}$.

$$
y_{j}^{(\tau)}=1 / \tau \sum_{i=(j-1) \tau+1}^{j \tau} x_{i} \quad 1 \leq \mathrm{j} \leq N / \tau
$$

Here, $s$ is the scale factor, and $L$ is the length of $x^{i}, y_{j}^{s}$ means the $j^{\text {th }}$ vector of $y$ with a certain $s$ (For example, $y_{3}^{5}$ is the $5^{\text {th }}$ vector when the scale factor is 3 ). The main idea is that the system is composed of distinguishable pieces or grains. And here we use coarse-grained process of time series to analyze the EEG data.

\section{EEG DATA}

This paper uses ECG data extracted from 12 persons when they are closing their eyes and idle in different period. The database has multi-parameter data, including 16 leads. We just extract Fp1 and Fp2 for research. The sampling frequency is $200 \mathrm{~Hz}$. 


\section{EXPERIMENT RESULTS}

The value of MSTE on closing eyes and being idle differs in the $\delta$ rhythm, when the scale factor is larger than
7, it is shown in the Fig. 1. And the difference is most significant when the scale is 18 . Therefore, we select the scale factor as 18 for further research.

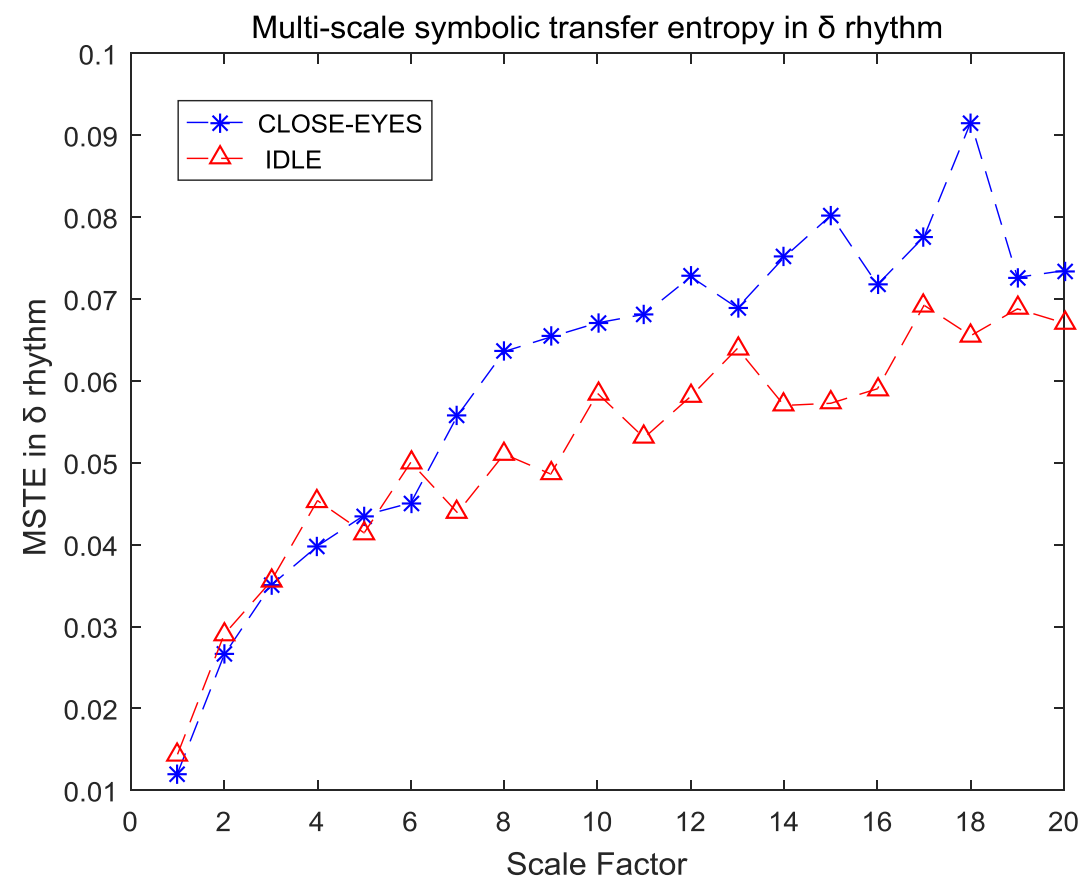

Figure 1. The value of MSTE on closing eyes and being idle in the $\delta$ rhythm

The mean and variance of the value of MSTE on closing eyes and being idle in the $\delta$ rhythm with scale factor as 18 is shown in the Fig. 2 .

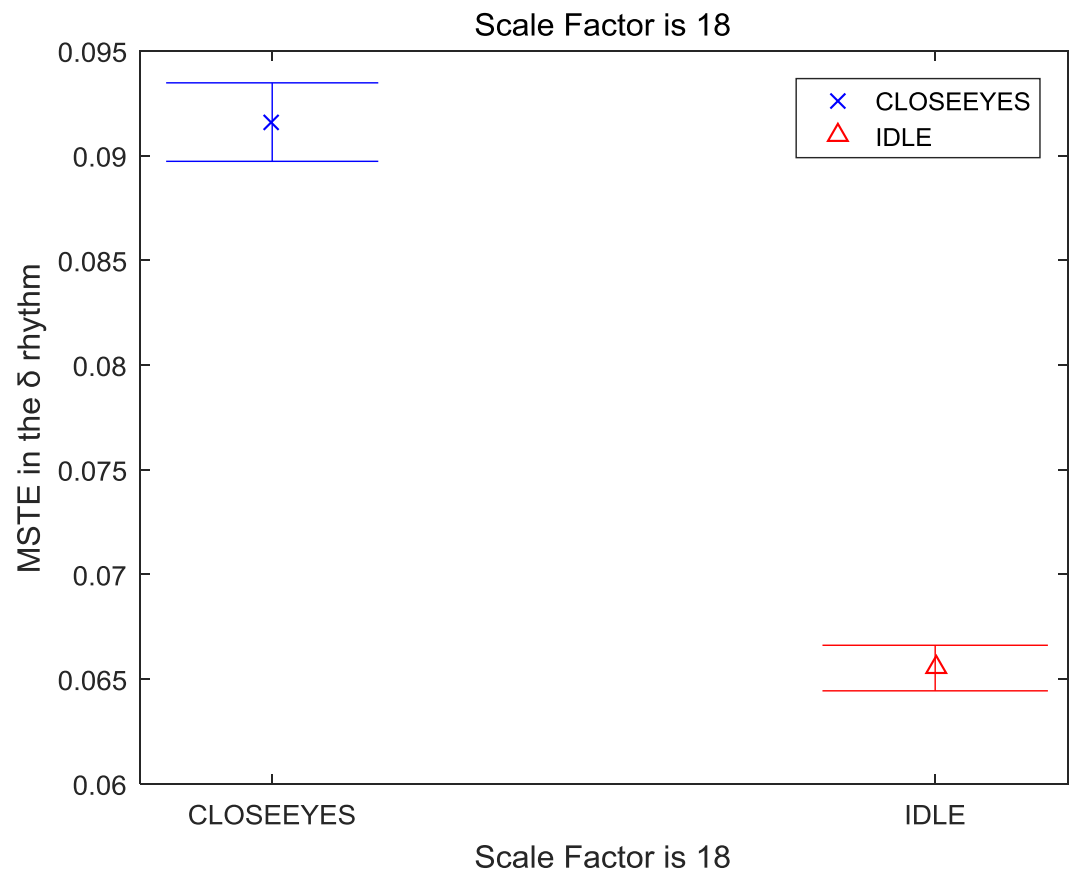

Figure 2. The mean and variance of MSTE in the $\delta$ rhythm as scale factor is 18

As we can see, the difference is significant and therefore we do the independent-samples T test for it.

We have 24 groups for each state (closing eyes or being idle). According to the value of $\mathrm{F}$, we can see that the result is 0.025 , lesser than 0.05 . Therefore, we can draw the conclusion that the different is significant and we then can say that the value of MSTE on closing eyes and being idle in the $\delta$ rhythm differs finally. 


\section{CONCLUSIONS}

MSTE is a useful, practical and effective tool to deal with EEG data. That is why we use it in this work. And finally we have found out that the value of MSTE on closing eyes and being idle in the $\delta$ rhythm differs. Here we have found out the exactly rhythm of EEG instead of the whole data. It is to say that our research is more accurately and useful in health care and medical treatment. However, the work could not quantize this difference and take it into practical use right now. Therefore, a further research is of great importance and we will try to make it.

\section{ACKNOWLEDGMENTS}

Project supported by the project is supported by the National Natural Science Foundation of China (Grant Nos. 61271082, 61401518, 31671006), Jiangsu Provincial Key R \& D Program (Social Development) (Grant No.BE2015700, BE2016773), the Natural Science Foundation of Jiangsu Province (Grant No. BK20141432),Natural Science Research Major Program in Universities of Jiangsu Province (Grant No.16KJA310002).

\section{REFERENCES}

[1] J. Kurths, A. Voss, P. Saparin, A. Witt, H.J. Kleiner, N. Wessel, Quantitative analysis of heart rate variability, Chaos 5 (1) (1995) 88-94.

[2] J. Li, X.B. Ning, Dynamical complexity detection in short-term physiological series using base-scale entropy, Phys. Rev. E (3) 73 (5 Pt 1) (2006) 052902.

[3] N. Wessel, C. Ziehmann, J. Kurths, U. Meyerfeldt, A. Schirdewan, A. Voss, Short-term forecasting of life-threatening cardiac arrhythmias based on

[4] C. Bandt, B. Pompe, Permutation entropy: a natural complexity measure for time series, Phys. Rev. Lett. 88 (17) (2001) 174102.

[5] Costa M, Goldberger AL, and Peng CK. Phys. Rev. Lett., Multiscale entropy analysis of complex physiologic time series, 2002; 89: 068102-1; 